\title{
AN APPLICATION OF ASSEMBLY STRUCTURE IN WELDING ESTIMATE OF WELDING OBJECT IN MANUFACTURING DESIGN
}

\author{
Zhihua Zhang", I. Nagasawa "*, M. Mochizuki", H. Yamaguchi ${ }^{* * *}$ and M. Umeda" \\ "Sanyo Women's College, ""Kyushu Institute of Technology, "** Choryo Design Ltd. Co., Japan
}

\begin{abstract}
Welding vessel objects such as heat-exchanger, oil-tank and other civil-used pressure vessels are comparatively large size products. Design works for such products not only concern with the geometric data, more important aspect is to treat with the design knowledge used in design process. But traditional CAD systems generally depend on shape-oriented approaches and pay less attention on the features of design and dealing with design knowledge. Therefore, there has been a considerable growth of interest toward knowledge-based design support systems. We have proposed a design object model for manufacturing design of welding vessels. In this model, the assembly structure of a design object is represented by the essential design elements and their dependencies. This paper discusses its application and focus on welding estimate of a welding object and dealing with design knowledge related.
\end{abstract}

Key words: Knowledge-based CAD, Design Object Model, Welding Estimate.

\section{INTRODUCTION}

In recent years, considerations of product development are remarkably increased such as the globalization of business, environmental problems, corresponding to new technologies, etc. Especially in the design business of welding vessels, it is mainly concerned about getting high reliability and low cost for high competition in a competitive worldwide manufacturing market, but it is very difficult to make a harmonized demand on quality, performance, economic efficiency and safety in design and production. So, how to improve the intelligent productivity of design work becomes more urgent. Till then, division and standardization on design activities are advanced in this field. Also for design automation, problems-oriented design support systems have 
been developed. But most of them directly coded the design procedures for particular product type in system structure. Modification is need whenever the design-plan changes because lack of integrated management abilities of design information. Therefore, we proposed a design object model, assembly structure [1], which centrally manages the design information such as cutting, welding, assembling, etc., and construction of design support system based on the model has also been discussed [2]. This paper focuses on welding estimate of a welding object and dealing with the related design knowledge.

\section{DESIGN OBJECT MODEL}

Welding vessel objects such as heat-exchanger, oil-tank and other pressure vessels are comparatively large size products. Generally, a welding vessel object is composed from tubes or/and globular body with nozzles, which usually manufactured by cutting, bending and welding steel plates or halfproduct parts like flanges. Much production is an article-ordered production with high performance and high reliability, also must fills to decided laws and safety standards. The design flow of such a product is usually divided as concept design, basic design, manufacturing design phases in our research.

\subsection{Assembly Structure}

In this model, the assembly structure of a design object is represented as assembly objects and their dependencies. It is able to centrally manage the design information generated in whole design process with related model.

- Assembly Object described by combination of Functional Elements based on the essential design operation units designed by designers, which not only include the form information of object but a designer who performed correlation with semantic processing or a functional operate in design.

- Dependency Relation used to describe the dependencies between assembly objects, such as part-of, dimension setting, machining process, etc.

In design, functional elements do interaction with others to embody its function and generally operated as a conceptual entity than form feature. An assembly object has attributes to identify its characteristics in design.

\subsection{Assembly Structure Module}

The stepwise refinement feature of design operation has been considered in the model. This is also helpful to represent the objects, for example, the welding lines. In design, a designer does it in a manner of succeeding 
determination. That is to say, it is firstly assigned as an empty-structured assembly object but owns design meaning, and then to be substituted alternatively by the compound assembly object that has a concrete assembly structure. We called it Assembly Structure Module. It is generally dealt with equally as a functional element and can be implemented as an abstract class from a viewpoint of software. The detailed structure of a module is determined in accordance with the progress of design operation.

\section{OPERATION OF ASSEMBLY STRUCTURE}

The assembly structure of a design object is constructed by using the following three types of design operations.

- Primitive Operation is a series of operations for constructing the data structure of a design object, editing the attributes of an assembly object and the dependencies between assembly objects in design.

- Basic Operation is the design operations related to manufacturing such as the end-cutting, hole-making and grooving, etc. of assembly objects.

- Macro Operation is a combination operation of primitive operations and basic operations to complete a series of design operations automatically if it is necessary.

\section{APPLICATION OF ASSEMBLY STRUCTURE}

Several applications of assembly structure model are available such as generation of manufacturing documents and 3D design information. Here, we focus on welding estimate of vessels because it is important for evaluate a design plan from aspects of cost and necessary for product makers.

Welding Estimate is to calculate the required cost of a welding vessel before manufacturing such as man-hours and material expense. Generally, long time is required for the job because there are many parts and processing points of a product, especially in case of large size. In many cases, a designer analogises by the actual achievements and experiences and diagrammatise it in a general view to support estimate. For example, by making the graph of model patterns and weight relation of a product, the estimate of alike product can be done by reverse reference of the graph. Although this method can estimate quickly, but cannot take out an exact estimate especially the product constitution changed. To solve the problem, as an application of assembly structure model, it is sufficient to manage manufacturing information such as man-hours related to cut processing, grooving and welding processing of a product into design models as the attributes of related object. By tracing the 
objects and evaluating the design attributes, the estimate can be sufficient done. Furthermore, factors like structure complexity and size of a design object, the estimate on man-hours of cutting and welding process is related to the design knowledge, which are systematically arranged according to different processing methods, especially for the public standards and the actual design know-how of specific factory. Design knowledge is deal with specific tools, which provide designers a simple knowledge process language based on the concept of data flow and some solutions search methods.

\section{CONCLUSION}

Traditional problem-oriented CAD systems mainly depend on shapeoriented approaches and pay less attention on the features of design operation and how to deal with design knowledge used in design process. We proposed the Assembly Structure, which provide a framework to centrally manage the design information in whole process. And the application of the model on welding estimate has been discussed. By adopting the techniques, systems can not only promote the intellectual productivity of design business, but also can cope with the new design more flexibly in product development. As future subject, it is necessary to systematize the design knowledge of welding vessel objects such as welding and structure standards that used in actual works. Additional research subject is to develop the related knowledge base for support system and test the ideas and approaches by construct a welding estimate support system so as to get the overall evaluation.

\section{REFERENCE}

1. Z. Zhang, I. Nagasawa, M. Mochizuki, H. Yamaguchi and M. Umeda: $A$ Design Object Model for Manufacturing Design of Welding Vessel Objects, Transactions of IPSJ, Vol.41, No.1, pp123-135 (2000).

2. Z. Zhang, I. Nagasawa, M. Mochizuki, H. Yamaguchi and M. Umeda A Design Support System for Manufacturing Design of Welding Vessel ObjectsThe Proceedings of IFIP $16^{\text {th }}$ WCC on ITBM-2000 pp.658-666 (August 2000)

3. Jami J. Shah and Mary T. Rogers: Assembly Modeling as a Feature-Based Design, Research in Engineering Design (USA), Vol.5, No.3-4, pp.218- 237 (1993).

4. V. Allada and S. Anand: Feature-based modeling approaches for integrated manufacturing: state-of- the-art survey and future research direction.Int. J. Computer Integrated Manufacturing, Vol.8, No.6 pp.411-440 (1995).

5. Japan Standard Society: the JIS handbook, Weld (1992). 